\title{
Public Knowledge and Attitudes towards Cross Infection and Infection Control in Dentistry during COVID -19 Pandemic
}

\author{
Mai E Khalaf $^{1 *}$, Afnan Faridoun ${ }^{2}$, Sarah A. Alkandari ${ }^{3}$, Qoot Alkhubaizi ${ }^{4}$
}

\author{
${ }^{1}$ DMD, MA, Associate Professor, Department of General Dental Practice, Faculty of Dentistry, Health Sciences Centre, Kuwait \\ University, Jabryia, Hawalli, Kuwait \\ ${ }^{2}$ DDS, MA, Assistant Professor, Department of General Dental Practice, Faculty of Dentistry, Health Sciences Centre, Kuwait \\ University, Jabryia, Hawalli, Kuwait \\ ${ }^{3}$ DDS, Dentist, Department of Bioclinical Sciences, Faculty of Dentistry, Health Sciences Centre, Kuwait University, Jabryia, Hawalli, \\ Kuwait \\ ${ }^{4}$ BSc. MS, Assistant Professor, Department of General Dental Practice, Faculty of Dentistry, Health Sciences Centre, Kuwait \\ University, Jabryia, Hawalli, Kuwait
}

DOI: $\underline{10.36347 / \text { sjds.2022.v09i01.002 }}$

| Received: 22.11.2021 | Accepted: 25.12.2021 | Published: 11.01.2022

*Corresponding author: Mai E Khalaf

Abstract

Original Research Article

Background: The aim of this study was to assess the knowledge of dental office environment during the pre- and postCOVID-19 pandemic, and attitude towards seeking dental treatment and care in a sample of participants who have access to dental care. Materials and Methods A structured 42 question survey questionnaire was utilized as the study instrument. The survey addressed three areas of knowledge regarding infection transmission in dental clinics, infection control measures used in dental clinics, and general knowledge about COVID-19. Descriptive statistics were presented. Associations between knowledge and attitude scales and demographic characteristics were evaluated. Results A total of 757 participants completed the survey. The study found that around one half of the respondents before the start of the COVID-19 pandemic would be regular visitors to the dentists. Approximately half reported visiting the dental clinic during the COVID-19 outbreak. Respondents reported the main sources of information they used to obtain information about the COVID-19 pandemic from official websites. There was a positive relationship between higher income and educational levels and greater knowledge concerning infection transmission in dental clinics and general knowledge about COVID-19. A significant positive relationship was found between the level of knowledge concerning infection transmission in dental clinics and agreement with the additional control measures taken during the pandemic. Conclusions The study population did not have an accurate understanding of the routes of transmission of the COVID-19 virus yet had a fair amount of knowledge about general infection control measures. Poor knowledge was associated with an increased perceived sense of security regarding seeking dental treatment among respondents.

Keywords: Infection control, Dentistry, COVID-19.

Copyright $\odot 2022$ The Author(s): This is an open-access article distributed under the terms of the Creative Commons Attribution 4.0 International License (CC BY-NC 4.0) which permits unrestricted use, distribution, and reproduction in any medium for non-commercial use provided the original author and source are credited.

\section{INTRODUCTION}

The magnitude of attention that the COVID-19 disease obtained globally is substantial, due to the unprecedented challenges and the detrimental effects it has posed worldwide. Consequently, the operation of healthcare facilities around the world has drastically adjusted to mitigate the risk of spreading the disease and to protect patients, healthcare providers and auxiliary staff. Many health care providers started offering virtual appointments and minimizing in-person interactions with patients. However, due to the unique nature of dental care, the presence of patients physically in the office was often necessary. In March 2020, the American Dental Association (ADA) recommended that all dental treatment, except for urgent and emergency care should be postponed. However, in November 2020 the ADA stated that "At this point in time, the American Dental Association (ADA) firmly believes dental care can continue to be delivered safely" and that "guidance recommended by the ADA and the Centers for Disease Control and Prevention (CDC) continues to safeguard the health of the public". This 
decision was based on a study that estimated the prevalence of COVID-19 among dentists in the United States to be less than $1 \%$. The conclusion of the study was that the current infection control measures and the use of enhanced personal protective equipment might be sufficient to prevent the spread of the virus in the dental setting [1].

The dental clinic environment is highly associated with biological hazards, which can inherently generate a level of concern among the public $[2,3]$. The close contact with patients, risk of exposure to saliva, blood, aerosols, as well as handling sharp instruments are all factors that places the dental practice in the high-risk category for transmission of different infectious agents [4,5]. As a matter of course, infectious agent-transmission in the dental office has been a longstanding concern among patients, and fear of contracting diseases has been driving some of them away from receiving the necessary treatment, even before the current outbreak of COVID-19 [3, 6]. Viral hepatitis, tuberculosis, and HIV, among others, all carry a risk of being transmitted in a dental clinic setting [3]. These infectious agents can be transmitted in the dental clinic via multiple routes such as contaminated surfaces, direct contact with an infected person, airborne and droplet spread [5]. The literature has also demonstrated that the dentist's attire could be contaminated with a plethora of contaminants [2].

The mode of transmission of the SARS-CoV-2 virus has become an emerging universal concern among the public in this current pandemic [3]. Dental Care Workers are at higher risk of contracting COVID-19 infection compared to other professionals [7]. This resulted in many patients electing to defer or refrain from dental treatment due to the highly aerosolizing and infectious environment of the dental office [8]. Although the risk of transmission within the dental office is high, there is no clear relationship between dental care and COVID-19 infections [9]. Still, in a survey carried out during the peak of COVID-19 outbreak, more than $79 \%$ of the sample perceived their risk of contracting COVID-19 infection through visiting dental clinics as moderate to high risk [10].

Although the pandemic is expected to plateau, SARS-CoV-2 virus will remain present with potential new variants. It is imperative that society and service delivery adapts to the new normal. With that, patients who abstained from dental care due to fear of contracting COVID-19, will have to resume their dental treatment. Patients who have poor knowledge regarding modes of transmission of SARS-CoV-2 were found to be fearful of receiving dental treatment [6]. This places an onus on dental professionals to educate the patient population about the dental office environment and the profession's infection control measures that are optimized and universally instituted to protect the patient and dental personnel.
The aim of this study was to assess the knowledge of dental office environment during the preand post-COVID-19 pandemic, and attitude towards seeking dental treatment and care during the COVID-19 in sample of participants who have access to dental care. can readily access dental care.

\section{MATERIALS AND METHODS Ethical considerations}

This study received ethical approval by the Health Sciences Center (HSC) Ethical Committee, University in full accordance with World Medical Association Declaration of Helsinki. Approval number VDR/EC/3787. The participants' confidentiality was maintained as neither the name nor the participants' contact information was involved in the questionnaire. All participants received electronic information about the nature and purpose of the study, and an electronic written informed consent was obtained from each subject upon their approval to participate. Participants were ensured that responses were anonymous, and participation was voluntary.

\section{Study design, study subjects, and study instrument}

A descriptive, cross-sectional survey was carried out from March to May 2021 among adult individuals throughout using a convenience sampling process. Inclusion criteria were adults 18 years of age or older who spoke Arabic or English, and who were able to consent to participation. Healthcare providers, healthcare students, and those who were unwilling to participate were excluded from the study. A structured, self-administered questionnaire was utilized as the study instrument. The questionnaire consisted of 42 close-ended questions, provided in both Arabic and English languages, that were particularly designed for the purposes of this study. Demographic data included in the questionnaire were age, gender, marital status, educational level, occupational status, and income level. The survey questions were categorized under main domains for knowledge: 1) infection transmission in dental clinics, 2) infection control measures used in dental clinics, and 3) general knowledge about COVID19 and attitudes: 1) additional infection control measures taken in dental clinics during the COVID-19 pandemic, and 2) feeling safer with dental staff using safety precautions. To assess the validity of the prepared questionnaire, a pilot survey was carried out among 20 adults. Subjects in the pilot study were excluded from the main study sample.

\section{Sample size and data collection}

Based on the population size of $3,255,261$ above 18-year-old in and using a $95 \%$ confidence level, $4 \%$ confidence interval, the power analysis calculation based on population size revealed a need for 601 respondents. Considering a $40 \%$ response rate due to the nature of the data collection the targeted sample size was 1503 respondent. 
With $99.5 \%$ of the population in owning a smart phone with internet, using social media platforms allowed access to most of the target population [11]. The data collection was completed over the course of sixty days. Utilizing Google forms, electronic copies of the questionnaire were uploaded and randomly distributed by broadcasting a text message and WhatsApp ${ }^{\circledR}$ link to all phone contacts that were collected through professional and private networks. Only completed surveys were permitted to be submitted, and the forms with identical IP addresses were not accepted ensuring no duplication of responses.

\section{Data analysis}

The Statistical Package for Social Sciences Software (SPSS) version 25 was used for data analysis. Descriptive statistics were presented using frequency, percentage, mean/average, and standard deviation (SD). For the knowledge and attitudes questions, correct answers were scored as " 1 ", and incorrect as " 0 ". Scores were analyzed as continuous variables in subsequent analyses. The associations between the knowledge and attitude scores and demographic characteristics were evaluated using Spearman's rank correlation coefficient and Mann-Whitney $U$ tests. Furthermore, Spearman's rank correlation coefficient test was used to assess the association between the sum of scores of the knowledge and attitude domains and income and education of the participants. A critical probability value ( $P$-value) of $<0.01$ was used as the cut-off level for statistical significance.

\section{RESULTS Participants}

Completed and eligible questionnaires were submitted by 757 participants. The majority $(81.2 \%)$ of the respondents were females, and the average age was 39.5 years \pm 11.8. Additional demographic characteristics of the participants are presented in Table 1.

About half the respondents reported that they visit the dentist regularly, and about half also said they visited the dental clinic during the COVID-19 outbreak. Almost one-half $(46.6 \%)$ of those visits were for emergencies or pain (Table 2).

When examining the factors that most likely influenced patients to seek dental treatment during the pandemic, dental check-up was the most likely procedure to be perceived as safe by the participants during the COVID-19 pandemic; $51.4 \%$ of the respondents reported they would feel comfortable receiving a check-up during the pandemic. The procedure that was perceived to hold the highest risk, by the greatest percentage $(38.3 \%)$ of respondents was scaling. Over $30 \%$ of the participants also reported that they would not feel safe receiving extractions or prosthodontic treatment during the pandemic (Figure 1).

\section{Knowledge and attitude domains}

Sixty five percent of participants answered questions relating to their general knowledge of infection transmission in dental clinics correctly, 75\% answered questions assessing their knowledge of infection control measures in dental clinics correctly. When asked about their knowledge of the transmission of the COVID-19 virus in dental clinics only $52 \%$ of the questions were answered correctly. Ninety two percent of respondents agreed with the use of additional measures to control infection during the pandemic and $82 \%$ agreed that they felt safer with dental personnel using optimized infection control precautions. These results are further illustrated in Figure 2.

Respondents' self-reporting of the main sources of information they used to obtain information about the COVID-19 pandemic showed that nearly half of the participants reported that they usually obtain general information on COVID-19 from varying official websites online. Furthermore, over a quarter of the participants demonstrated that they rely on social media to get such information (Table 3 ).

\section{Correlations between knowledge and attitude scores and demographics}

The correlations of income and educational levels shown in Table 4 indicate significant positive relationships between higher income and educational levels and greater knowledge concerning infection transmission in dental clinics, and general knowledge about COVID-19. There was a positive relationship between the level of education and participants' knowledge of the infection control measures used in dental clinics $(p=0.012)$. In addition, a negative correlation between income and attitudes toward the use of additional control measures during the pandemic indicated that those control measures were less important to participants with higher incomes ( $p<$ $.001)$.

Gender comparisons indicate that females reported greater knowledge concerning infection transmission in dental clinics $(p=.006)$. Females were also significantly more in favor of using additional control measures during the pandemic compared to males $(p=.013)$.

Only one difference was observed between participants who were married versus those who were single, divorced, or widowed. Married participants were significantly less knowledgeable regarding the infection control measures used in dental clinics $(p=.003)$.

Participants who were employed reported greater knowledge concerning infection transmission in dental clinics $(p=.004)$ and higher general knowledge about COVID-19 $(p<.001)$. On the other hand, those who were unemployed were significantly more likely to endorse the use of additional control measures during 
Mai E Khalaf et al., Sch J Dent Sci, Jan, 2022; 9(1): 6-13

the pandemic $(p=.024)$ and were also more likely to report feeling safer when observing the dental staff using safety precautions $(p=.004)$.

\section{Correlations between knowledge and attitude domains}

Table 5 presents the associations between the knowledge and attitude total domain scores and employment. A significant positive relationship $(p<$ $.001)$ was found between the level of knowledge concerning infection transmission in dental clinics and agreement with the additional control measures taken during the pandemic. A significant negative correlation $(p<.001)$ was found between knowledge of infection transmission in dental clinics and attitude toward feeling safer with dental staff using safety precautions. No significant relationships were observed between the other two knowledge domains and the attitude domains.

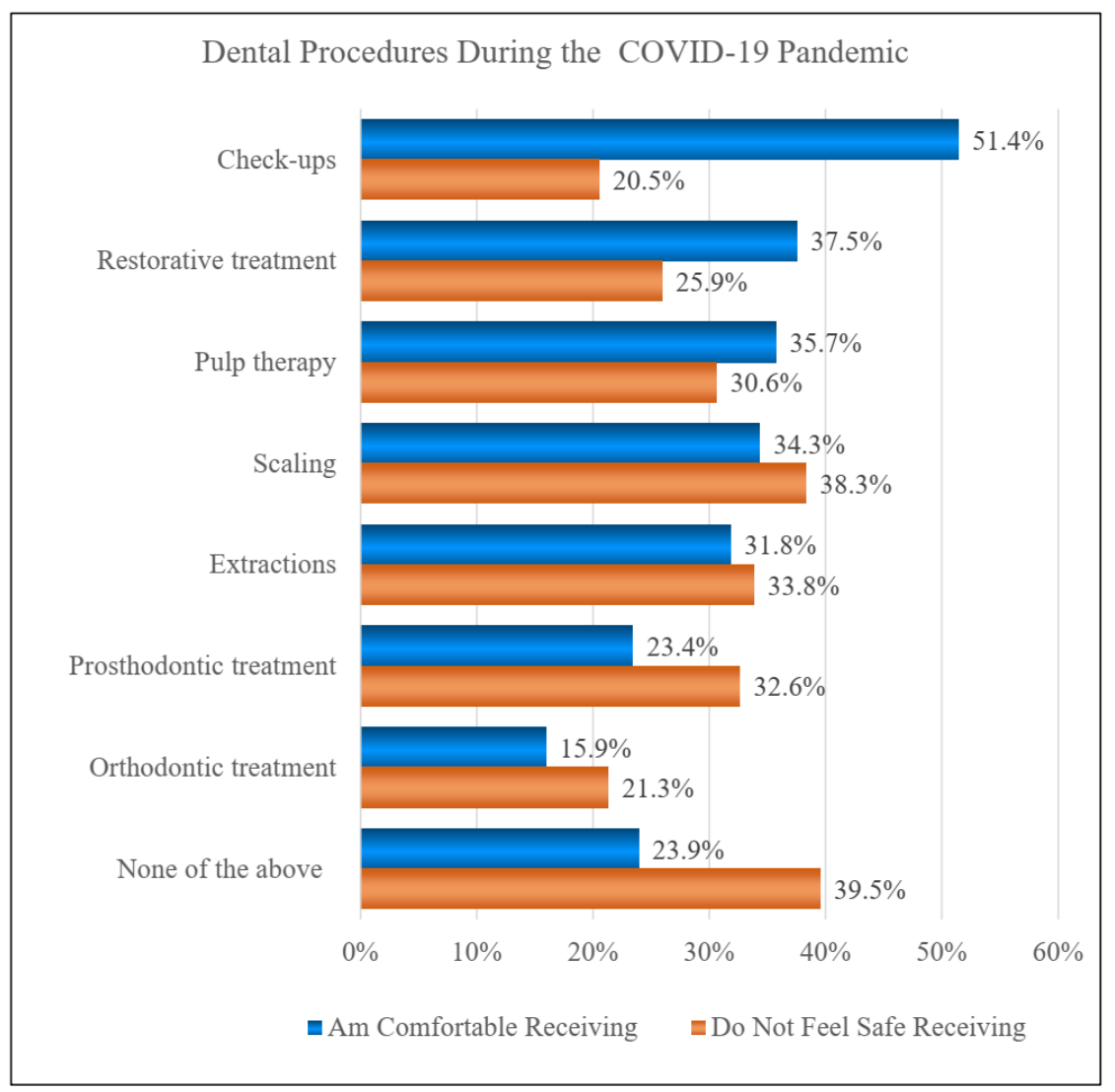

Figure 1: Comfort and discomfort with receiving dental procedures during the COVID-19 pandemic

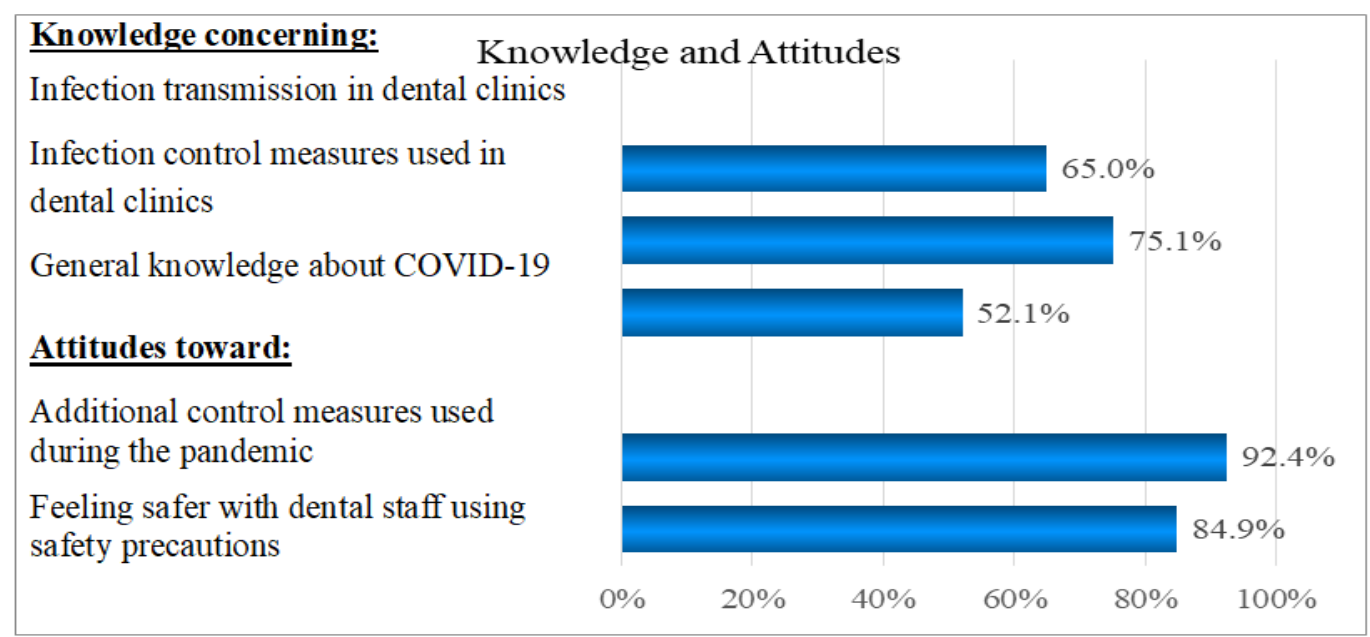

Figure 2: Average Percentages of correct knowledge and percentage of positive attitudes 
Mai E Khalaf et al., Sch J Dent Sci, Jan, 2022; 9(1): 6-13

Table 1: Demographic characteristics of the participants $(\mathbf{n}=757)$

\begin{tabular}{|l|l|l|}
\hline \multicolumn{2}{|l|}{ Frequency } & Percent \\
\hline Gender & 615 & 81.2 \\
\hline Female & 142 & 18.8 \\
\hline Male & \multicolumn{2}{l|}{} \\
\hline Marital status & 217 & 28.7 \\
\hline Single & 467 & 61.7 \\
\hline Married & 63 & 8.3 \\
\hline Divorced & 10 & 1.3 \\
\hline Widowed & \multicolumn{2}{l|}{} \\
\hline Education & 78 & 10.3 \\
\hline High school or less & 501 & 66.2 \\
\hline University Bachelor degree & 178 & 23.5 \\
\hline Graduate degree (Masters, PhD) & 204 & 26.9 \\
\hline Employed & 553 & 73.1 \\
\hline No & 163 & 21.5 \\
\hline Yes & 158 & 20.9 \\
\hline Income level & 285 & 37.6 \\
\hline$<500$ KD & 151 & 19.9 \\
\hline $500-999$ KD &
\end{tabular}

Table 2: Dental visits

\begin{tabular}{|l|l|l|}
\hline \multicolumn{3}{|l|}{} \\
\hline Visit dentist regularly & Frequency & Percent \\
\hline No & 377 & 49.8 \\
\hline Yes & 380 & 50.2 \\
\hline Visited dental clinic during COVID-19 outbreak \\
\hline No & 353 \\
\hline Yes & 404 & 46.6 \\
\hline Reason: & 170 & 53.4 \\
\hline Emergency/Pain & 78 & 42.1 \\
\hline Check-up & 70 & 19.3 \\
\hline Scaling & 43 & 17.3 \\
\hline Restorative treatment & 43 & 10.6 \\
\hline Orthodontic treatment & 10.6 \\
\hline
\end{tabular}

Table 3: Main sources of information on COVID-19

\begin{tabular}{|l|l|l|}
\hline & Frequency & Percent \\
\hline Formal/official websites & 369 & 48.7 \\
\hline Social media & 200 & 26.4 \\
\hline Doctor/dentist & 94 & 12.4 \\
\hline Television/radio & 66 & 8.7 \\
\hline Family/friends & 13 & 1.7 \\
\hline Books/magazine & 9 & 1.2 \\
\hline Non-formal websites & 3 & 0.4 \\
\hline Other & 3 & 0.4 \\
\hline
\end{tabular}

Table 4: Spearman correlations between income and educational levels and the knowledge and attitude questions

\begin{tabular}{|c|c|c|c|c|}
\hline & \multicolumn{2}{|c|}{ Income } & \multicolumn{2}{|c|}{ Education } \\
\hline & rho & $p$ & rho & $p$ \\
\hline \multicolumn{5}{|l|}{ Knowledge concerning: } \\
\hline Infection transmission in dental clinics & 0.25 & $<.001$ & 0.10 & 0.008 \\
\hline Infection control measures used & 0.09 & 0.012 & 0.07 & 0.065 \\
\hline General knowledge about COVID-19 & 0.30 & $<.001$ & 0.22 & $<.001$ \\
\hline \multicolumn{5}{|l|}{ Attitude toward: } \\
\hline Use of additional control measures during the pandemic & -0.07 & 0.075 & -0.14 & $<.001$ \\
\hline Feeling safer with dental staff using safety precautions & -0.04 & 0.261 & 0.02 & 0.615 \\
\hline
\end{tabular}


Mai E Khalaf et al., Sch J Dent Sci, Jan, 2022; 9(1): 6-13

Table 5: Mann-Whitney $U$ tests on the knowledge and attitude questions by employment

\begin{tabular}{|l|l|l|l|l|l|l|l|}
\hline & Employed & & \\
\hline & Yes $(\mathbf{n}=\mathbf{5 5 3})$ & No $(\mathbf{n}=\mathbf{2 0 4})$ & & \\
\hline & Mean & SD & Mean & SD & $z^{*}$ & $\boldsymbol{p}$ \\
\hline \multicolumn{7}{|l|}{} \\
\hline Knowledge concerning: & 66.76 & 26.25 & 60.29 & 28.22 & 2.90 & 0.004 \\
\hline Infection transmission in dental clinics & 75.56 & 13.75 & 73.94 & 13.95 & 1.50 & 0.133 \\
\hline Infection control measures used & 53.91 & 21.31 & 47.18 & 20.64 & 3.81 & $<.001$ \\
\hline General knowledge about COVID-19 & 91.71 & 14.95 & 94.28 & 12.92 & 2.26 & 0.024 \\
\hline Attitude toward: & 84.20 & 11.60 & 86.86 & 11.91 & 2.88 & 0.004 \\
\hline Use of additional control measures during the pandemic
\end{tabular}

*standardized Mann-Whitney $U$ test

\section{DISCUSSION}

It has been reported that psychological implications of fear and anxiety are natural in pandemics, especially when the morbidity and mortality rates continue to increase [12]. The present study aimed to seek the levels of knowledge and attitudes among the general population towards seeking dental care before and during the COVID-19 pandemic.

Most of the respondents in this study were married females with a university degree and of middleclass income levels. This demonstrates that the trends and thoughts that are reported in this paper represent the middle-class level of the society who are educated and have access to information through multiple sources of media.

The current study found that around one-half of the respondents, prior to the onset of the COVID-19 pandemic, would be regular visitors to the dentists. A similar proportion of the respondents, around $47 \%$, reported that they visited the dentist since the beginning of the pandemic. These findings are found to be in alignment with a report that investigated the trends in visiting the dental office in the United States during the pandemic. This study demonstrated that there was a substantial rebound in the rates of weekly dental visits by June 2020. This came after a major decline during the early phases of the pandemic, which can be attributed to the increase in knowledge about the nature of transmission and preventive measures [13].

The notable finding was that when asked about the reason for their visits, the majority reported emergencies or dental pain as the reason for their visits. This can be attributed to the fact that at the beginning of the pandemic, many of the health centers operated on an emergency basis only, thus providing care for patients with severe odontogenic infections and pain. However, this is not the sole factor contributing to this since the survey distribution was done several months after the ease of the lockdown restrictions, and once most national and private dental centers started administering routine dental care. Contrary to other regions worldwide, it was found that in Beijing China, there has been a decrease in the numbers of patients seeking emergency dental treatment due to the fear of potential risk of infection by going outdoors [14]. In North America, it has also been reported that dental emergency visits have declined as a significant number of patients received antimicrobial therapy prior to emergency appointments via tele dentistry consultations, reducing the actual patient visits to clinics [15]. Furthermore, it has been shown that during the spring of 2020, nearly half of the participants of a survey in the United States reported that they have intentionally delayed their dental treatment due to the pandemic [16]. This may not have been observed in our case as in Kuwait, where the present study was conducted, the option of tele dentistry is not one that is readily available to the general population. In Kuwait, dental and health services are nationalized and are available to all Kuwaiti nationals and resident nonKuwaitis through primary and tertiary care centers all over the State of Kuwait. Availability of health services is abundant with 72 primary care centers hosting a team of health care professionals scattered in the various administrative districts of Kuwait. In addition, another study conducted in Saudi Arabia demonstrated that only $8.8 \%$ of the Saudi population had visited their dentists during the COVID-19 outbreak, with pain being the most common reason for those visits [17].

A group of researchers in Italy categorized dental treatment based on the risk of transmission of the COVID-19 virus. This was achieved through creating a risk-scoring system where 42 common dental procedures were classified into three risk categories being low, medium or high and suggested a safety protocol in accordance with the risk of the category. Radiographic examinations and diagnostic procedures were allocated a low-risk score while scaling was considered in the high-risk category [18]. In this study, with regards to procedures that patients are continuing to seek dental care for as life resumes since the start of the pandemic, checkups were perceived as the safest dental procedure and that they felt most comfortable receiving. Dental scaling was reported as the least safe and therefore reported as less likely to be sought. Similar results were found in another study conducted among Caucasian adults in Spain, in which routine dental check-ups were perceived as the least risky dental procedures [19]. 
It has been reported worldwide that there will be a general fear of visiting dentists among the public after the COVID-19 outbreak settles down [20]. Fear of contracting COVID-19 was reported to be a significant deterrent from seeking dental care [10]. Our study assessed patients' knowledge of infection transmission and infection control in dental offices of general diseases and in relation to COVID-19. The study also evaluated patients' perceived attitudes towards safety and infection control measures that they observe during their dental visits. Overall, although participants of this study did not have an accurate understanding of COVID-19 routes of transmission (with only 52\% correctly responding to general knowledge questions about COVID-19), they did have a fair idea of general infection control methods. Interestingly, we found that when respondents had less overall knowledge about infectious disease transmission, they tended to have an extra sense of safety in dental offices. Comparably, in King Abdulaziz University Hospital, Jeddah, Saudi Arabia, a group of researchers assessed the knowledge of their patients about infectious diseases and assessed their attitude towards dental treatment. The results showed that most of their respondents displayed poor $(39.5 \%)$ to fair $(38.7 \%)$ knowledge about infections and infection control in dentistry yet demonstrated a positive attitude towards seeking treatment at the dental office [21].

As is widely understood, to address factors such as overall population knowledge and attempt to increase it, there has to be an understanding of what the most common sources of information that the study population seeks in their daily lives. The major source reported by the research population was formal and official (governmental and health authorities) websites. This was followed by social media posts. In social networking applications such as Instagram, Snap Chat, WhatsApp and TikTok are popular and heavily utilized in disseminating local news and medical information. Many medical professionals use these platforms for educating members of the society and use influencers to endorse their health messages. This finding is in accordance with other studies carried out among the Saudi population, which reported that the website of the Ministry of Health was the preferred source of information on COVID-19 followed by social media [21]. These findings overemphasized the positive role and impact of the governments in addressing the pandemic situation.

The conducted study had some limitations, as the data was collected in the current time period with the pandemic still ongoing, the practices continue to evolve to accommodate that. Therefore, it is expected that patients' attitudes and knowledge may alter more rapidly as more information is relayed to them. The study was also conducted online, with a female majority, and therefore information gathered reflected the knowledge and attitudes of a large proportion society who are literate. Therefore, limiting the overall representativeness of the findings. In order to be more generalizable and provide a better understanding of the trends alternative methods need to be explored to gather information from the sectors of the population who do not have access to things such as the internet or network television, etc.

\section{CONCLUSIONS}

The results of this research demonstrated that the study population did not have an accurate understanding of the routes of transmission of the COVID-19 virus yet had a fair amount of knowledge about general infection control measures. Additionally, poor knowledge was associated with an increased perceived sense of security regarding seeking dental treatment among respondents. Attitudes in the public towards seeking dental care in a pandemic are expected to improve with the continued understanding of the disease course, enhancement of dental personal protective equipment (PPE) in the dental office and increase in vaccination rates.

\section{Disclosure Statement}

The authors of this publication declare that they have no conflict of interest. The authors did not receive institutional support for this publication.

Data Availability Statement: All data generated or analyzed in this study are included in the article.

\section{REFERENCES}

1. Estrich, C. G., Mikkelsen, M., Morrissey, R., Geisinger, M. L., Ioannidou, E., Vujicic, M., \& Araujo, M. W. (2020). Estimating COVID-19 prevalence and infection control practices among US dentists. The Journal of the American Dental Association, 151(11), 815-824.

2. Anders, P. L., Drinnan, A. J., \& Thines, T. J. (1998). Infectious diseases and the dental office. New York State Dental Journal, 64(4), 2934.

3. Pasiga, B. D. (2020). Relationship Knowledge Transmission of COVID-19 and Fear of Dental Care During Pandemic in South Sulawesi, Indonesia. Pesquisa Brasileira em Odontopediatria e Clínica Integrada, 21, e0148.

4. Al-Amad, S. H., Awad, M. A., Edher, F. M., Shahramian, K., \& Omran, T. A. (2017). The effect of rubber dam on atmospheric bacterial aerosols during restorative dentistry. Journal of infection and public health, 10(2), 195-200.

5. Ashtiani, R. E., Tehrani, S., Revilla-León, M., \& Zandinejad, A. (2020). Reducing the Risk of COVID-19 Transmission in Dental Offices: A Review. Journal of Prosthodontics, 29(9), 739-745.

6. Peng, X., Xu, X., Li, Y., Cheng, L., Zhou, X., \& Ren, B. (2020). Transmission routes of 2019-nCoV and controls in dental practice. International journal of oral science, 12(1), 1-6. 
7. Sonica Singhal, B. D. S., Warren, C., Hobin, E., \& Smith, B. (2021). How Often Are Dental Care Workers Exposed to Occupational Characteristics that Put Them at Higher Risk of Exposure and Transmission of COVID-19? A Comparative Analysis. J Can Dent Assoc, 87(116), 1488-2159.

8. Nazir, M., Almulhim, K. S., AlDaamah, Z., Bubshait, S., Sallout, M., AlGhamdi, S., \& Alhumaid, J. (2021). Dental Fear and Patient Preference for Emergency Dental Treatment Among Adults in COVID-19 Quarantine Centers in Dammam, Saudi Arabia. Patient preference and adherence, 15, 1707-1715.

9. Banakar, M., Bagheri Lankarani, K., Jafarpour, D., Moayedi, S., Banakar, M. H., \& MohammadSadeghi, A. (2020). COVID-19 transmission risk and protective protocols in dentistry: a systematic review. BMC oral health, 20(1), 1-12.

10. Ibrahim, M. S., Alibrahim, H., Al Madani, A., Alamri, A., Bamashmous, M., \& Tounsi, A. (2021). Fear Factor in Seeking Dental Care among Saudis during COVID-19 Pandemic. International Journal of Environmental Research and Public Health, 18(20), 10589.

11. https://www.citra.gov.kw/sites/en/Pages/ict_indicat ors.aspx

12. Ahmed, M. A., Jouhar, R., Ahmed, N., Adnan, S., Aftab, M., Zafar, M. S., \& Khurshid, Z. (2020). Fear and practice modifications among dentists to combat novel coronavirus disease (COVID-19) outbreak. International journal of environmental research and public health, 17(8), 2821.

13. Kranz, A. M., Chen, A., Gahlon, G., \& Stein, B. D. (2021). 2020 trends in dental office visits during the COVID-19 pandemic. The Journal of the American Dental Association, S00028177(21)00138-0.
14. Guo, H., Zhou, Y., Liu, X., \& Tan, J. (2020). The impact of the COVID-19 epidemic on the utilization of emergency dental services. Journal of dental sciences, 15(4), 564-567.

15. Wu, K. Y., Wu, D. T., Nguyen, T. T., \& Tran, S. D. (2021). COVID-19's impact on private practice and academic dentistry in North America. Oral Diseases, 27, 684-687.

16. Kranz, A. M., Gahlon, G., Dick, A. W., \& Stein, B. D. (2021). Characteristics of US adults delaying dental care due to the COVID-19 pandemic. JDR Clinical \& Translational Research, 6(1), 8-14.

17. Srivastava, K. C., Shrivastava, D., Sghaireen, M. G., Alsharari, A. F., Alduraywish, A. A., AlJohani, K., ... \& Alzarea, B. K. (2020). Knowledge, attitudes and practices regarding COVID-19 among dental health care professionals: a cross-sectional study in Saudi Arabia. Journal of International Medical Research, 48(12), 0300060520977593.

18. Bizzoca, M. E., Campisi, G., \& Lo Muzio, L. (2020). An innovative risk-scoring system of dental procedures and safety protocols in the COVID-19 era. BMC Oral Health, 20(1), 1-8.

19. González-Olmo, M. J., Ortega-Martínez, A. R., Delgado-Ramos, B., Romero-Maroto, M., \& Carrillo-Diaz, M. (2020). Perceived vulnerability to Coronavirus infection: impact on dental practice. Brazilian Oral Research, 34, e044.

20. Barabari, P., \& Moharamzadeh, K. (2020). Novel coronavirus (COVID-19) and dentistry-A comprehensive review of literature. Dentistry journal, 8(2), 53.

21. Ibrahim, N. K., Alwafi, H. A., Sangoof, S. O., Turkistani, A. K., \& Alattas, B. M. (2017). Crossinfection and infection control in dentistry: Knowledge, attitude and practice of patients attended dental clinics in King Abdulaziz University Hospital, Jeddah, Saudi Arabia. Journal of infection and Public Health, 10(4), 438-445. 